ROL DEL ESTADO Y LA POLÍTICA FISCAL EN COLOMBIA 1990- 2010*

Role of the state and fiscal policy in Colombia 1990-2010

\author{
Luisa Carrillo Corrales ${ }^{1}$ \\ María Paz Silva González ${ }^{1}$ \\ Amaury Jiménez Martínez²
}

\title{
RESUMEN
}

Uno de los principales factores del nivel bienestar económico y de renta per cápita de una economía es la política fiscal. El tamaño del sector público, las distintas funciones del gasto público y la estructura fiscal a través de la cual se financia el sector público inciden en las decisiones económicas y en la actuación de los entes privados. Lo que se pretende con este trabajo es presentar un análisis del papel del Gobierno en la economía y de la política fiscal durante el periodo corrido de apertura económica comprendido entre 19902010, desde una perspectiva que permita considerar las distintas cuestiones que se han suscitado en la literatura durante las últimas dos décadas, discutiendo las principales conclusiones en el terreno teórico.

Palabras claves: Gobierno, Política Fiscal, Transferencias.

Clasificación JEL: N4, E61, E62

\footnotetext{
* Documento presentado en las Jornadas de Investigación de la Facultad de Ciencias Económicas de la Universidad de Cartagena

${ }^{1}$ Programa de Economía de la Facultad de Ciencias Económicas- Universidad de Cartagena

${ }^{2}$ Docente del Programa de Economía de la Facultad de Ciencias Económicas- Universidad de Cartagena
} 


\begin{abstract}
One of the main factors of economic welfare level and per capita income of an economy is fiscal policy. The size of the public sector, the various functions of public spending and tax structure through which the public sector affect economic decisions and the actions of private entities is financed. The aim of this paper is to present an analysis of the role of government in the economy and fiscal policy during the run of economic openness comprised between 1990-2010 period, from a perspective that allows us to consider the various issues that have arisen in literature over the past two decades, discussing the main findings on theoretical grounds.
\end{abstract}

Key words: Government, Fiscal Policy, Transfers

JEL Clasification: N4, E61, E62

\title{
INTRODUCCIÓN
}

Es importante preguntarse ¿qué o quién es el Estado? De alguna manera se tiene cierta idea de cuáles son las instituciones que lo conforman: en el caso colombiano, el Congreso de la República, el Senado, la Corte Suprema de Justicia, el Gobierno nacional, departamental, municipal y una multitud de instituciones y organismos públicos autónomos. Los países occidentales tienen una estructura descentralizada de gobierno, es decir poseen varias esferas de actuación del Estado: la local, la provincia, la regional o autonómica, según los casos, y la central.

El Estado, a través del Gobierno es responsable de la defensa nacional, de la regulación del comercio nacional e internacional, de la educación, de la asistencia social, de la justicia, del cuidado de la calidad del ambiente, del suministro de los servicios públicos y de la provisión de servicios locales, como bibliotecas, alcantarillado y recolección de basuras. Aunque constitucionalmente suele establecerse a qué esfera de gobierno corresponde los derechos no mencionados explícitamente, los límites exactos son, por lo general, ambiguos.

¿Por qué ambiguos? Existen algunas veces en que no está clara la frontera entre las instituciones públicas y las privadas. Cuando el Gobierno crea una sociedad mercantil, una empresa pública, ¿forma 
ésta parte del "Estado"? Por ejemplo, El caso de Ecopetrol, donde el Estado es el principal accionista, pero no es el único, ya que también hay participación accionaria de particulares.

Entonces ¿qué diferencia a las instituciones que hemos llamado Estado de las privadas? Según Stigliz (2000), dos importantes características. "Primero, en una democracia las personas responsables de dirigir las instituciones públicas son elegidas o nombradas por otra persona elegida (o nombrada por otra persona que es nombrada por otra que es elegida...). La legitimidad de la persona que ocupa el cargo emana, directa o indirectamente, del proceso electoral. En cambio, los responsables de la administración de General Motors, son elegidos por los accionistas de la compañía y los responsables de la administración de fundaciones privadas (como Rockefeller o la March), por un patronato permanente.

En segundo lugar, el Estado posee determinados derechos de coerción de los que carecen las instituciones privadas ${ }^{3}$.De aquí que el Estado es una parte trascendental para cualquier comunidad organizada. Éste se puede definir como el conjunto de instituciones políticas, leyes y prácticas a través de las cuales se realiza la actividad de gobernar y se regula el funcionamiento de la sociedad.

Los principales objetivos del Estado, a través de las diferentes instituciones que lo conforman, en las sociedades modernas son: proteger las libertades individuales, velar por el cumplimiento de las normas y promover el bienestar de la sociedad como un todo; tomando en cuenta esos objetivos, es fácil inferir que el Estado desempeña un papel muy importante en una sociedad, tanto desde el punto de vista político como desde el punto de vista económico. A menudo al Estado se le denomina también sector público, debido a que las actividades que ejerce están al servicio de la comunidad o de la nación.

Si nos concentramos en su dimensión económica, el Estado es uno de los principales agentes que participan en el sistema económico junto con los hogares y las empresas1; sin embargo, el gobierno tiene una característica distintiva: como veremos más adelante, no sólo adelanta labores productivas

\footnotetext{
${ }^{3}$ Por ejemplo, tienen derecho a obligarnos a pagar impuestos (y, en caso contrario, a embargar nuestras propiedades y/o a encarcelarnos). Tiene derecho a expropiar nuestras tierras para uso público. Las instituciones privadas y los particulares no sólo carecen de estos derechos, sino que, además, el Estado restringe el derecho de los individuos a conferir a otros poderes de coerción similares (Stiglitz, J. La economía del sector público, p.21).
} 
como las empresas, y desarrolla actividades de consumo como las familias, sino que además debe garantizar que las reglas del juego de la economía se cumplan.

Para alcanzar sus objetivos, el Estado debe, por una parte, contar con un importante volumen de ingresos y, por otra, decidir cuál es la mejor manera de gastar esos recursos de acuerdo con los intereses de la sociedad. La mayor parte de los ingresos que recibe el Estado se derivan de los impuestos directos e indirectos que cobra a los hogares y a las empresas.

La decisión de cómo gastar esos ingresos se lleva a cabo en una instancia política que busca que en ese proceso se vean representados los intereses de los distintos sectores que conforman la sociedad, y aquella se consigna en el presupuesto del gobierno.

Las decisiones que toma el gobierno, como parte del Estado en cuanto a sus ingresos y su gasto con el fin de incidir sobre el desempeño de la economía se denominan política fiscal ${ }^{4}$. Estas se pueden clasificar en dos grupos: políticas expansionistas o políticas contraccionistas. La política fiscal "expansionista" se presenta cuando se toman medidas que generen aumento en el gasto del gobierno, o reducción de los impuestos, o una combinación de ambas. Por el contrario, una política fiscal "contraccionista" se presenta cuando se toman decisiones para tener un gasto gubernamental reducido, o aumentar los impuestos, o una combinación de ambas.

En medio del debate entre los que critican al gobierno por intervenir demasiado en la economía y los que reclaman una mayor participación pública en la vida económica del país, surgen algunas preguntas importantes: ¿Cuál debe ser el papel del gobierno en la economía? ¿Hasta dónde es responsabilidad del gobierno en el desarrollo de ciertas actividades productivas? ¿Es el gobierno un aliado de los hogares y los empresarios o, por el contrario, es una carga para su buen desempeño económico? ¿Cuáles son los instrumentos con que cuenta el gobierno para estimular la actividad económica de un país, y cuáles son las precauciones que debe tener en su manejo?

\footnotetext{
${ }^{4}$ Fiscal viene de la palabra "fisco" que significa ‘tesoro del Estado'; es decir, el tesoro público, el que nos pertenece a todos.
} 
La investigación pretende abordar los conceptos fundamentales relacionados con la participación del gobierno en la economía, y analiza la política fiscal durante el periodo de inicio de la apertura económica en la administración Gaviria (1990), hasta el 2010, fecha de terminación del gobierno de Álvaro Uribe Vélez. Esta discusión permite entender las circunstancias bajo las cuales un gobierno puede ejecutar la política fiscal de manera responsable y sostenible, así como los riesgos que existen cuando las autoridades ejecutan acciones que a primera vista lucen razonables, pero que pueden terminar siendo nocivas para la economía y la comunidad.

El documento presenta en cinco partes incluyendo esta introducción. La segunda parte incluye el marco teórico y presenta una breve revisión a la literatura relativa a la temática abordada de la Política fiscal y el rol del Estado. La tercera parte aborda el rol del estado y la política económica. Una cuarta parte, Las finanzas públicas en la última década del siglo XX y primera década del siglo XXI y por último se presentan las conclusiones.

Cabe aclarar que el presente documento aporta al análisis teórico de la política fiscal y su manejo en la economía colombiana, en ningún momento se modela datos ni se hace uso de series estadísticas para pronósticos o predicciones de cambios relativos en su manejo.

\section{MARCO REFERENCIAL}

\section{Marco Conceptual}

Para alcanzar sus objetivos, el Estado debe, por una parte, contar con un importante volumen de ingresos y, por otra, decidir cuál es la mejor manera de gastar esos recursos de acuerdo con los intereses de la sociedad.

Las decisiones que toma el gobierno, como parte del Estado en cuanto a sus ingresos y su gasto con el fin de incidir sobre el desempeño de la economía se denominan Política Fiscal. Fiscal viene de la palabra "fisco" que significa 'tesoro del Estado'; es decir, el tesoro público, el que nos pertenece a todos.

Desde el punto de vista del ingreso, el Estado puede controlar a quién y en qué cantidad se le cobran impuestos, a la vez que puede desarrollar mecanismos para garantizar el pago de éstos (evitar la 
evasión), etc. mas desde el punto de vista del gasto, el Estado puede tener influencia sobre el monto de partida presupuestaria que se transfiere a cada dependencia Estatal, el nivel de los salarios, el aumento de éstos año tras año, las contrataciones y los dineros que se transfieren a los estados y municipios o a otras entidades, etc.

La política fiscal está llamada a jugar un papel importante en el desarrollo económico de un país, se resaltan tres aspectos que pueden ser influenciados por la política fiscal: el crecimiento económico, la distribución del ingreso y las posibilidades de crecimiento y desarrollo, mediante su incidencia en la estabilidad macroeconómica.

Desde el punto de vista macroeconómico, la caracterización de la política fiscal envuelve tres elem entos primordiales: i) su postura frente al ciclo económico; ii) si es fuente de volatilidad macroeconómi ca, y iii) su sostenibilidad en el mediano y largo plazo. Con estas tres dimensiones, usualmente se dice que una "buena política fiscal" es aquella que es contracíclica (o acíclica, en su defecto), que no es fuen te de volatilidad macroeconómica y que es sostenible en el tiempo (Fatas y Mihov, 2009)

\section{Marco Teórico}

La teoría clásica se aferraba a que el sistema económico era capaz de alcanzar y mantener los niveles de ocupación deseados sin necesidad de la intervención del gobierno. Entre algunos de los economistas que compartían el pensamiento clásico se encuentran: Smith, Malthus y David Ricardo, los cuales defendían la propiedad privada, los mercados y creían que solo a través del principio de la competencia tiene la economía política una pretensión de ser ciencia. Y los neoclásicos explicaban la formación de los precios, no en función de la calidad de trabajo sino en función de la intensidad de la preferencia de los consumidores. Con ello se ampliaron conceptos macroeconómicos que más tarde se consolidaron en materia económica.

A partir de la década de 1870, los economistas neoclásicos como William Stanley en Gran Bretaña, León Walras en Francia, y Karl Menger en Austria, imprimieron un giro a la economía, abandonaron las limitaciones de la oferta para centrarse en la interpretación de las preferencias de los consumidores en términos psicológicos. 
John Maynard Keynes rompió decididamente con la escuela clásica del pensamiento económico, de la que el mismo era producto, lanzo una nueva teoría que habría de producir lo que más tarde se habría conocido como la revolución Keynesiana.

La posición de Keynes en relación con el papel que debía jugar la política fiscal parte del hecho de que su defensa por la intervención pública sólo se justifica en situaciones de desequilibrio. Los fundamentos básicos de la política fiscal keynesiana son la negación de la norma clásica del equilibrio presupuestario, la reducción de la brecha entre el Producto Bruto Interno real y el potencial, el papel del sector público centrado en incrementar el gasto privado (consumo e inversión); es decir, estabilizar la economía, pero no de una forma provisional ni transitoria, sino permanentemente y la eliminación de los problemas derivados de la existencia de un sistema tributario flexible (estabilizadores automáticos), que en las fases de crecimiento elevado recauda más disminuyendo automáticamente las rentas del sector privado, mediante el establecimiento de mecanismos de devolución al sector privado de este exceso de recaudación cuando la economía se encuentre alejada de su nivel de pleno empleo.

Por otro lado, Friedman y Los monetaristas son escépticos respecto a la utilidad de la política fiscal para controlar la demanda agregada y se oponen a los gastos del gobierno considerando que tienen poca efectividad, pues simplemente desplazarán la demanda privada de inversión; en otras palabras, lo único que lograrán los gastos gubernamentales será sustituir la iniciativa privada por la pública.

Sólo la política monetaria puede influir el curso del PIB. Detrás de este razonamiento existe un argumento que ya hemos escuchado: los aumentos en el gasto público sólo desplazan al gasto privado: el gobierno gasta más, pero los hogares o las empresas gastan menos. Se dice que éste es el caso debido a que el gobierno tiene que financiar su actividad mediante fijación de impuestos o usando préstamos y que éstos restringen la capacidad del sector privado para gastar por su propia cuenta.

Como consecuencia de esto, los monetaristas desacreditan la política fiscal y alaban la política monetaria. Llevando a un extremo, el lema del monetarismo que no es sólo que "el dinero importa" sino que "sólo el dinero importa". 


\section{Estado Del Arte}

Diversos autores han dado sus consideraciones respecto a las acciones ejercidas y pretensiones con la política fiscal de un país. Doménech (2004) considerando los efectos que la política fiscal debe ejercer en el bienestar social concluye para España, entre otras cosas que la política fiscal no parece afectar de forma permanente las tasas de crecimiento de la renta per cápita sino de forma transitoria, por lo que sólo tiene efectos importantes sobre el nivel de renta a largo plazo, así mismo que debe seguir avanzándose en la mejora de la medición de las variables fiscales, en particular de los tipos impositivos efectivos que gravan las distintas rentas de los factores productivos y el consumo, y de la productividad de los servicios públicos.

En cuanto a trabajos que estudian la política fiscal en Colombia estos se focalizan en detectar la naturaleza estructural del déficit fiscal encontramos a Lozano y Aristizábal (2003) que examinan el impacto de la política fiscal sobre la actividad económica desde 1990 hasta 2002 por medio de ejercicios de impulso respuesta. Estos ejercicios permitieron demostrar que un incremento de los gastos de consumo del Gobierno se traduce en un aumento positivo y significativo del PIB.

Lozano (2009) concluye en sus investigaciones que la caracterización macroeconómica de la política fiscal en Colombia en el largo plazo sustenta tres rasgos importantes: (i) que ésta ha sido históricamente pro-cíclica; (ii) que ha sido consecuente con la condición de sostenibilidad de la deuda en el largo plazo y (iii) que la política discrecional del gobierno ha reducido su volatilidad en los últimos años, sin dejar de lado que estas características de las acciones fiscales en Colombia también se han encontrado en las principales economías de la Unión Europea, aunque en grado diferente y con importantes diferencias entre los países que hacen parte de la Unión.

\section{El rol del estado y la política económica}

Todas las sociedades tienen que resolver algunos problemas económicos fundamentales que hacen referencia a: Asignar los recursos escasos, distribuir los ingresos, asegurar la estabilidad económica y darle dinámica al proceso económico. Estas funciones básicas de toda organización social tienen solución de formas distintas, dependiendo del tipo de estructura económico y política que tenga el Estado. En economías socialistas, es el Estado quien da respuesta a estos problemas económicos, por el 
contrario, en las economías capitalistas, estos problemas se resuelven mediante el libre juego del mercado.

Existen, otras formas de estructuras económicas que combinan las dos formas anteriores para dar respuesta a esos problemas (economías mixtas). El Estado colombiano hace parte de este tipo de organización económica. Algunas de estas funciones tienen mayor prioridad en un sistema que en otro. Así el capitalismo tiende a asignar los recursos de manera más eficiente económicamente, a través del mercado, pero genera una mayor inequidad en la distribución del ingreso.

Adam Smith en su obra Naturaleza y causas de la riqueza de las naciones (1776) ${ }^{5}$ habla sobre el papel del sector público en un sentido restrictivo, algo que era de esperar si se tiene en cuenta su pensamiento acerca de las ventajas del Mercado libre, del sistema de producción privado y la defensa de los derechos de propiedad.

"El primer deber del soberano es proteger a la sociedad de la violencia y la invasión de otras sociedades independientes, y eso puede ser logrado sólo por medio de la fuerza militar. Pero el gasto en preparar esa fuerza militar en tiempos de paz y de usarla en tiempos de guerra es muy diferente en distintos tipos de sociedad. "6

Por otra parte, se plantea que los gastos militares aumentan según el grado de evolución de la sociedad: son insignificantes en una sociedad de cazadores, bajos en una sociedad de pastores y muy altos en una sociedad industrial, pero constituyen un deber esencial del soberano (Estado).

"El segundo deber del soberano es el de proteger todo lo posible a cada miembro de la sociedad de la injusticia o de la opresión de sus restantes miembros. "7

Es decir que la segunda función del Estado es brindar a los individuos justicia y seguridad. Por último,

\footnotetext{
${ }^{5}$ Ver Smith, A., The wealth of nations, The University of Chicago Press, Chicago, 1976 (edición original, An inquiry into the nature and causes of the wealth of nations, 1776) (hay traducciones al español: Investigación sobre la naturaleza y causas de la riqueza de las naciones, Fondo de Cultura Económica; La riqueza de las naciones, Alianza).

${ }^{6}$ Ibídem, Volumen II, p.213.

${ }^{7}$ Ibídem, Volumen II, p. 231.
} 
"El tercer y último deber del soberano o de la república es el de fundar y mantener aquellas instituciones públicas y aquellos trabajos públicos que, aunque sean en el mayor grado ventajosos para una sociedad en su totalidad, son sin embargo de tal naturaleza que la ganancia no puede retribuir nunca el gasto de cualquier individuo o grupo de individuos, y que por ello no puede esperarse de ninguna persona o grupo de personas que lo funden o mantengan." 8

\section{Principios de Política Económica}

Un principio general de la teoría económica fue plantado por J. Tinbergen ${ }^{9}$, quien sostenía que si se desea garantizar que un conjunto de metas de política económica sean alcanzables, se necesita por lo menos que existan un número igual de instrumentos. Además, existe el principio de "eficiencia de los instrumentos", el cual dice que cada instrumento debe emplearse en aquella meta en la cual es más eficiente. Tinbergen también distingue entre objetivos y metas de la política económica. En los primeros ubica propósitos últimos de la política económica, verbi gracia, el "bienestar general" de la comunidad, mientras que entre los segundos incorpora propósitos más concretos a través de los cuales se cumple el objetivo final de la política económica. Entre éstos se resaltan los siguientes: La mejor distribución del ingreso o mayor equidad, la eficiencia en la asignación de recursos, el crecimiento económico y la estabilización económica ${ }^{10}$

La política fiscal emplea como instrumentos los tributos, el gasto y la deuda pública. En la meta de distribución del ingreso se utilizan al impuesto como mecanismo para gravar al rico y al gasto público como instrumento para beneficio de las clases económicas más necesitadas. En la estabilización económica, la herramienta fiscal puede utilizarse como regulador de la oferta y de la demanda agregada, ya que cuando hay inflación se utilizan instrumentos contraccionistas y cuando hay recesión se aplican instrumentos expansionistas. Los primeros implican aumentos en impuestos, disminución en el gasto público, incrementos de la deuda pública interna y cancelación de la externa, para el segundo caso, se hace lo contrario. La política fiscal también se utiliza para lograr equilibrar la balanza de pagos, para solucionar el problema de déficit debe emplearse políticas contraccionistas y el superávit se remedia mediante instrumentos expansionistas, dentro de ciertos límites.

8 Ibídem, Volumen II, p. 244.

9 Véase, Tinbergen (1968). Política económica, México, FCE, pp. 59-70.

10 Ibídem, pp. 72-81. 
Por otro lado, la formación de capital es una meta que puede ser coadyuvada con instrumentos de política fiscal. Con el crecimiento ilimitado de la oferta de trabajo en Colombia y la escasa respuesta del sector productivo privado, los gobiernos se ven en la necesidad de dar solución al problema del desempleo $^{11}$. Según el DNP (1970), si se buscase mantener el desempleo absoluto constante, y si el crecimiento de la población es del 3,5\% anual y la relación marginal empleo-producto es de 1/2, el PIB deberá crecer al $7 \%$ anual, para lo cual se requeriría una inversión en capital físico del $21 \%$ del PIB (sobre el supuesto de una relación marginal producto-capital de 1/3). El país debe utilizar políticas fiscales con el fin de incrementar la tasa de crecimiento del PIB y mitigar el problema del desempleo.

A través de la Política Fiscal, el Estado cumple con tres grandes funciones:

1. Busca la reasignación de los recursos de la economía.

2. Redistribuye el ingreso

3. La estabilización macro y el estímulo al crecimiento económico de largo plazo. Estos tres aspectos contribuyen a mejorar el bienestar social.

Existen varias razones por las cuales se produce un déficit fiscal, entre estas razones se pueden destacar las siguientes:

- Aumento de las cargas tributarias: Cuando este fenómeno se presenta la primera medida que toma el gobierno es la de elevar los impuestos, lo que a su vez crea una disminución en los ingresos disponibles tanto industrial como personal, se disminuye la capacidad de inversión del sector privado, originando un incremento en las tasas de desempleo del país.

- Disminución de las tasas de crecimiento: Como se señaló en el punto anterior, la inversión del sector privado se ve afectada, generando desempleo, disminución de la demanda y el consumo, y estancamiento en el crecimiento económico.

La reducción en la inversión privada conlleva graves resultados para el país como una disminución en el ritmo de crecimiento, que a su vez no permite disminuir los niveles de desempleo. Para que la economía presente un ritmo de crecimiento más favorable, se hace necesario incentivar la inversión privada y crear una estrategia exportadora competitiva.

11 Lewis, W. A. (1954). Desarrollo económico con oferta ilimitada de trabajo, p.139. 
Existen otros efectos de la reducción de la inversión privada, tales como: altas tasas de interés, contracción del gasto público, disminución de la inversión privada y pública, y desigualdad distributiva del ingreso.

- Déficit en la cuenta corriente: El déficit fiscal y en la cuenta corriente de la balanza de pagos son considerados en la teoría macroeconómica como déficits gemelos, por la gran relación e incidencia que existe entre ellos. La principal vía por la cual se produce esta relación es la reevaluación de la tasa de cambio como respuesta a un mayor déficit fiscal, afectando también las áreas productivas. Éstas, a su vez, generan efectos adicionales como incremento en los niveles de endeudamiento, alza en la tasa de interés, disminución de los niveles de inversión pública y privada, desempleo y recesión.

- Inestabilidad en los Precios: Se asocian prolongados déficits fiscales con altas tasas de inflación. Esto se debe a los incentivos que tienen los gobiernos para financiar su déficit con emisión primaria. Sin embargo esta relación no es directa y deja de lado factores importantes como la disponibilidad que tienen los gobiernos para financiarse por otros medios diferentes a la emisión como pueden ser el crédito y el alza de impuestos variables y va aún más allá cuando se piensa que existen otras variables que afectan a los precios además de la cantidad de dinero en la economía como puede ser la tasa de cambio real, las prácticas de indexación de salarios, contratos, etc., y las expectativas de los agentes.

- Balanza de Pagos: Usualmente se cree que los desbalances fiscales dan origen a déficits en las balanzas comerciales de los países. Los canales de transmisión no son muy claros; algunos autores señalan que el aumento en los tipos de interés provocadas por la expansión del gasto ocasiona el ingreso de capitales y hacen que el tipo de cambio se aprecie lo que produce un deterioro en la balanza comercial. Otros autores advierten que los pagos hechos por concepto de intereses y amortizaciones perjudican la cuenta de capital y producen un desbalance en la balanza de pagos.

Por último, la política fiscal se puede utilizar como instrumento para la asignación de los recursos. Según el profesor Musgrave de coordinar la política de aranceles aduaneros y de impuestos a las ventas para evitar que la protección exagerada genere distorsiones en el proceso productivo ${ }^{12}$.

12 Gillis y McLure, Propuesta de una reforma fiscal para Colombia. Bogotá: Ediciones Banco de la República, 1974, Tomo III, pp. 29-57. 


\section{Las finanzas públicas en la última década del siglo xx y primera década del siglo xxi La administración Gaviria (1990-1994)}

El plan de desarrollo económico y social de la administración Gaviria, conocido con el nombre de La Revolución Pacífica (DNP, 1991), busca promover un ambiente institucional para que el sector privado, y en general todos los ciudadanos, sean los actores centrales del proceso de cambio y motor del crecimiento económico, dándole una mayor importancia relativa al funcionamiento de los mercados. El Estado puede hacer más por el desarrollo económico concentrándose en sus obligaciones sociales básicas, desvinculándose de funciones que no le corresponden, y dejando de intervenir en numerosos escenarios de la vida económica.

Los sucesos que se dieron en las economías socialistas, y el creciente consenso sobre la nueva orientación del Estado en la economía, han incidido en la conformación de las ideas de los políticos sino sobre las doctrinas, sobre el crecimiento y desarrollo económico. Las antiguas ideas que exaltaban la inversión física, el papel activista del Estado, el supuesto papel reactivador del gasto público, los sectores líderes y el proteccionismo a ultranza, han quedado relegadas conceptualmente, por su ineficiencia e inutilidad. Por el contrario, el papel de los mercados, la información, el efecto del capital humano y las externalidades son los temas presentes en las agendas para lograr el desarrollo.

La década del noventa en el país, se inició bajo un ambiente de crisis política e institucional que condujo a la convocatoria de una Asamblea Nacional Constituyente y a la expedición de una nueva Constitución Política en 1991. La opinión de los constituyentes, era que la nueva Carta Política permitiría recuperar la credibilidad en las instituciones, daría mayores mecanismos de participación directa al pueblo en la toma de decisiones y en general, haría más eficiente la administración pública.

En materia de descentralización fiscal la nueva constitución política, y posteriormente sus normas reglamentarias, aumentaron el volumen de transferencias del gobierno central hacia los departamentos y municipios y redefinieron las funciones de los distintos niveles de gobierno, aumentando las responsabilidades de las entidades territoriales. ${ }^{13}$

\footnotetext{
${ }^{13}$ En el campo político, se instauró la elección popular de gobernadores, el tarjetón, la financiación de campañas y las consultas populares en los niveles territoriales (Departamento Nacional de Planeación, 1991).
} 
Por otra parte, la reforma constitucional de 1991 consolidó el proceso y los principales cambios introducidos en ésta contemplaron: el establecimiento de un marco para redefinir las funciones de cada nivel de gobierno y de las diferentes categorías de departamentos, el fortalecimiento de los municipios al duplicarles en un período de diez años la proporción de los ingresos corrientes de la Nación transferidos, y la creación de un fondo con las regalías nacionales para inversiones en las regiones.

Los artículos 356 y 357 de la nueva Constitución Política de Colombia (1991) establecieron el marco general tanto para la asignación de funciones entre los gobiernos subnacionales, como para la distribución de las transferencias del gobierno nacional hacia las entidades territoriales. En particular, el artículo 356 definió el destino de los recursos del situado fiscal, dirigido a financiar la educación y la salud, y fijó las pautas para la distribución de estos recursos entre los gobiernos departamentales y los distritos especiales ${ }^{14}$. Así mismo, este artículo estableció que no se "podrán descentralizar responsabilidades sin la previa asignación de los recursos fiscales suficientes para atenderlas." Es importante anotar que en este artículo no se especifica el porcentaje de los ingresos corrientes de la nación que deben ser transferidos con este fin.

Por otra parte, el artículo 357 fijó los porcentajes sobre los ingresos corrientes de la nación que deben transferirse a los municipios y a otras entidades territoriales y estableció algunos criterios para su distribución. Si bien el artículo señala que las "participaciones" municipales se destinan a la inversión social, no definió los programas específicos que se financian con estos recursos. ${ }^{15}$ Estos artículos fueron reglamentados por la Ley 60 de 1993. Se estableció que las transferencias por situado fiscal debían ser del 23\% del total de los Ingresos Corrientes de la Nación (ICN) en 1994, para 1995, el 23,5\% y del 24,5\% de 1996 en adelante (ver Articulo 10 de la Ley 60 de 1993); mientras que las participaciones de los municipios ascenderían del 14\% de los ICN (1993) hasta lograr un mínimo del 22\% (2002) (Artículo 357 de la Constitución).

\footnotetext{
14 "El 15\% del situado se distribuirá en partes iguales entre los departamentos, el distrito capital y los distritos de Cartagena y Santa Marta. El resto se asignará en proporción al número de usuarios actuales y potenciales de los servicios de salud y educación, teniendo en cuenta, además, el esfuerzo fiscal ponderado y la eficiencia administrativa de la respectiva entidad territorial" (Constitución Política de la República de Colombia 1991, art. 356).

${ }^{15}$ El artículo contempla que: "la participación de los municipios se incrementará, año por año, del 14\% de los ingresos corrientes de la nación en 1993 hasta alcanzar el 22\% como mínimo en el año 2002.” Así mismo, el artículo estableció que "el 60\% de las participaciones se distribuirá en proporción directa al número de habitantes con necesidades básicas insatisfechas y al nivel relativo de pobreza de la población de cada municipio." El 40\% restante deberá distribuirse "de acuerdo a la población total, la eficiencia fiscal y administrativa y el progreso demostrado en calidad de vida" (Constitución Política de la República de Colombia 1991, Art. 357).
} 
En lo que respecta a la política fiscal, además de la reforma tributaria (Ley 49 de 1990) que incremento la tarifa del IVA del 10\% al 12\% y que amplió su base, los ingresos del Estado se vieron fortalecidos con las mejoras administrativas en la recaudación y con los ajustes en las tarifas de servicios públicos (Hommes, 1994). Esta reducción del déficit fiscal buscaba reducir la inflación en el país.

Al acelerarse el proceso de apertura económica a mediados de 1991, con la disminución de los recaudos aduaneros, el Gobierno se vio obligado a recurrir de nuevo al Congreso para solicitar nuevos aumentos en los impuestos (Ibíd.). El Congreso convino elevarlos del $12 \%$ al $14 \%$ y continuar aplicando la base (Ley 6 de 1992). Por otra parte, el gasto público consolidado revelo un aumento del 20,6\% en 1990, al 23,6\%, en 1993, mientras los del Gobierno aumentaron del 9,4\% del PIB, en 1990, al $12 \%$ del PIB en 1993.

\section{La administración Samper (1994-1998)}

Las bases de la estrategia económica de la administración Samper estaban formuladas en el Plan Nacional de Desarrollo: El Salto Social (DNP, 1995), donde el objetivo primordial era lograr mayores niveles de equidad y para ello la política fiscal jugaba un papel relevante.

Para 1995 se proyectaba un déficit del Gobierno del 2,9\% del PIB, que sería compensado con los excedentes del resto del sector público (Perry, 1995), todo esto debido a las apropiaciones de gasto que se habían ejecutado en la administración anterior, principalmente por el crecimiento de las transferencias territoriales (Constitución 1991) y pensiónales (Ley 100 de 1993).

El ministro Perry presentó ante el Congreso un proyecto de ley sobre racionalización tributaria, el cual fue aprobado mediante Ley 223 del 1 de diciembre de 1995, lo que logro aumentar la tasa de impuesto de renta para personas jurídicas de 30\% a 35\%, estableció una renta presuntiva sobre el patrimonio líquido del 5\%. El IVA aumento la tasa al 16\% y quito el IVA para algunos insumos de la construcción de vivienda.

Las proyecciones para 1996 mostraban un deterioro mayor en el balance fiscal, ya que los ingresos crecerían menos de lo esperado, sino también por que las apropiaciones de gasto presentaban un 
aumento superior al 30\% frente a la definitiva del año anterior (Ocampo, 1997). En efecto, el SPNF pasó de un superávit de 0,1\% del PIB (1994), a un déficit del 1,7\% (1996).

Los ingresos corrientes se incrementaron un 2\% del PIB (1990-1996), incluido el impacto de las reformas tributarias, mientras que los gastos los habían hecho un 5,5\% (Ibíd.). Esta situación se le atribuye a tres factores principales: las transferencias públicas ligadas con la Constitución Política de 1991, la incidencia de la Ley 100 de 1993 que había modificado los regímenes de pensiones y la Ley 4 de 1992 con sus impactos en aumentos de sueldos y remuneraciones a las fuerzas armadas. Durante el primer semestre de 1997 no se adoptaron medidas fiscales y la situación continuó deteriorándose (Robinson y Urrutia, 2007).

Por otra parte, la Comisión de racionalización del gasto estimó que las decisiones constitucionales de 1991 sobre transferencias del situado fiscal para educación y salud implicaron un incremento en el gasto de $1 \%$ del PIB, en tanto que por concepto de las transferencias a los municipios el gasto se incrementó en 0,7\% (Rosas, 1997). Otras decisiones constitucionales relacionadas con el fortalecimiento de la fiscalía, el sector justicia y el subsidio a tarifas de servicios públicos explica otro $0,5 \%$ del PIB, para un total de 2,2\% del PIB originado en gastos adicionales motivados por la Constitución del 1991.

\section{La administración Pastrana (1998-2002)}

En diciembre de 1999 se firmó un acuerdo entre el Gobierno de Colombia y el Fondo monetario Internacional (FMI), denominado Acuerdo extendido o de facilidades extendidas (1999-2002), por valor de 2.700 millones de dólares (equivalentes a $1.957 \mathrm{DEG})^{16}$.

El "desequilibrio fiscal" de 1999 se atribuye a la introducción de programas en los primeros años de la década de los años 90 que significaron grandes aumentos del gasto público (Banco de Republica), debido a las transferencias presupuestales para garantizar los derechos fundamentales de salud, seguridad y educación. De hecho, el gasto en educación paso de 3,49 billones a 5,1 billones y el de

\footnotetext{
${ }^{16}$ Así se denominan los derechos especiales de giro, que son las inversiones de los países en el FMI. No se recurrió a dichos recursos porque, según la Contraloría General de la Republica, "Estos acuerdos han sido, más bien, parte de la estrategia para el manejo de la política económica orientada a asegurar el acceso al mercado internacional de capitales y a los recursos de la banca multilateral como forma de garantizar el financiamiento de la economía y el pago de la deuda externa” (CGR, 2003, p.7).
} 
salud de 1,33 billones a 2,36 billones de 1994 a 1999. Para las autoridades económicas este fue la mecha que encendió la crisis, aunque en esos seis años apenas ese incremento significo alrededor de 2,5 billones (Sarmiento, 2005, pp. 376 y 378).

Las autoridades resaltan el aumento del gasto público y su ineficiente asignación como uno de los problemas centrales que enfrentaba la economía hacia finales de los noventa. Se destaca que el endeudamiento público se multiplico y alcanzó niveles exageradamente altos (Restrepo, 1999, 2000). En efecto la proporción de la deuda del Gobierno sobre el PIB pasó de 18\% en 1997 a 30\% en 1999. Este tema de la deuda se convirtió en centro de preocupación desde ese momento para los Gobiernos, las entidades multilaterales y los mercados.

Para hacer frente al déficit fiscal, el Memorando del Acuerdo Extendido del FMI trazo un programa de ajuste para el periodo 1999-2002, centrado en la consolidación fiscal, en la restructuración del sector financiero, en la flexibilidad de la tasa de cambio y en la implementación de reformas estructurales. En esta misma dirección, programo una baja en la inflación del 10 al 6\%, un crecimiento del PIB real para el 2000 al 3\% y llevarlo al 5\% en el 2002. En cuanto al déficit en cuenta corriente la meta fue de 1,3\% del PIB en 1999 y mantenerlo por encima del 3\% en 2001 y 2002. Para lograr este nivel de déficit se debía dar un financiamiento de mediano y largo plazo, más inversión extranjera directa, con esto se calculó que la "deuda externa colombiana se mantendría entre el 43 o 44\% del PIB (Banco de la República).

Para controlar el gasto público en el mediano plazo, se presentó una enmienda constitucional al Congreso en septiembre de 1999 con el objeto de mantener constante en términos reales el valor de los fondos que se transferían a los gobiernos locales bajo los acuerdos de participación en los ingresos corrientes de la Nación. En otras palabras, se planteó desvincular dichos traslados del crecimiento de los ingresos corrientes y amarrarlos a la inflación ${ }^{17}$.

\footnotetext{
${ }^{17}$ El Acto Legislativo de 2001 reformó los artículos 356 y 357 de la Constitución de 1991, al fusionar en el denominado sistema general de participación (SGP) las antiguas participaciones municipales en los ICN y el situado fiscal, desligando las cesiones nacionales al nivel territorial de los ingresos corrientes nacionales y hacerlas depender de la inflación (que está entre el 4 y $6 \%$ anual) más unos puntos adicionales por año, así: entre el 2002 y 2005, la inflación causada y el $2 \%$ anual; entre 2006- 2008, la inflación más 2,5\% cada año y a partir del 2009, el promedio de la variación de los ICN en los cuatro últimos años. De acuerdo con investigaciones del DNP, la Contraloría General de la República y el Ministerio de hacienda, en el periodo 2002-2005, la Nación ahorró al menos 3,7 puntos del PIB, ya que, mientras al inicio del periodo de transición, es decir, año 2001, el nivel territorial (departamentos, distritos y municipios) representó el 46,5\% de los ICN, con la fórmula del Acto Legislativo 1 de 2001, para el 2005 sólo representó el 37,2\% de los ICN
} 
El Memorando del FMI reconoció que ese cambio implicaría un ahorro del 0,4\% del PIB (2001) y del $0,8 \%$ (2002). Las medidas de política fiscal para lograr "una fuerte disminución del gasto del Gobierno central excluyendo el pago de intereses" (punto 16 del Memorando) se resume en el cuadro 1.

Cuadro 1. Medidas de política fiscal inscritas en el Memorando del Acuerdo Extendido del FMI (diciembre de 1999)

\begin{tabular}{|c|c|}
\hline Para aumento de los ingresos & Para disminuir el Gasto Social \\
\hline $\begin{array}{l}\text { Aumento de la base del IVA y del } \\
\text { impuesto de renta }\end{array}$ & $\begin{array}{l}\text { Reducción del tamaño del Estado. Despidos. Ajuste fiscal a entes } \\
\text { territoriales: Ley } 617 \text { de } 2000\end{array}$ \\
\hline Devaluación & Bajas alzas salariales y congelaciones de sueldos \\
\hline Sobretasa a la gasolina & Municipalización de la salud y la educación \\
\hline Bonos de paz & $\begin{array}{l}\text { Disminución de las transferencias para salud y educación. Acto } \\
\text { Legislativo 01-2001 y Ley } 120-2001\end{array}$ \\
\hline $2 \times 1.000$ permanente & Eliminación de regímenes especiales de pensiones \\
\hline $\begin{array}{l}\text { Fortalecimiento de } \\
\text { administración de impuestos }\end{array}$ & $\begin{array}{l}\text { Punto 16. "Una fuerte reducción del gasto del Gobierno central } \\
\text { con excepción del pago de intereses }\end{array}$ \\
\hline Privatizaciones & $\begin{array}{l}\text { Concesiones en infraestructura vial, férrea y aeroportuaria (el } \\
\text { Estado sale de la operación de estos sistemas) }\end{array}$ \\
\hline
\end{tabular}

Fuente: Suarez (2002, p. 34)

\section{La administración de Uribe Vélez (2002-2010)}

El lema que caracterizo los ocho años de gobierno del presidente Uribe Vélez. "Trabajar, trabajar y trabajar", tuvo como objetivo central consolidarlo sobre las bases construidas a partir del neoliberalismo implementado por la administración de Andrés Pastrana en los últimos años del siglo XX.

A finales de los años noventa, Colombia vivió una de sus mayores crisis económicas y sociales que llevó en 1999 a que el Producto Interno Bruto cayera un 4,2\%, la pobreza moderada se ubicara en niveles cercanos al 58\% y la pobreza extrema en el 25\% (DNP, 2008). Para mitigar el impacto social de la crisis se implementaron una serie de medidas como la puesta en marcha de una Red de Apoyo

(reducción efectiva del 9,3\%). Esto implicó para los entes territoriales una perdida entre 14 y 16 billones de pesos entre 2002 y 2005 (Reyes, 2007). 
Social, dirigida a las poblaciones más vulnerables para el fortalecimiento del capital humano de las familias y la inserción al mercado laboral.

Por otra parte, a partir de 2002 en Colombia se comenzó a implementar la Política de Seguridad Democrática (con la convicción de que sólo a través de incrementos en la seguridad y en la confianza tanto de los ciudadanos como de los inversionistas, se lograría dinamizar y profundizar el crecimiento incluyente y el desarrollo para todos), el saneamiento de las finanzas públicas y reducción del tamaño del Estado (Junguito, 2003).

Una vez posesionado el nuevo gobierno, se planteó el grave problema de las finanzas públicas y los desequilibrios sociales, por ello el Presidente anunció que los ingresos del Estado deben servir para ayudar a los más pobres, manifestando: "Los empresarios que con tenacidad sirven bien a la Nación, llevarán sobre sus hombros nuevas responsabilidades tributarias. Los esfuerzos de los sectores medios y populares deben retribuirse en mayor inversión social para frenar la renovación de su prolongado purgatorio".

De inmediato se empezó a pulir una propuesta de reforma tributaria que conciliara las propuestas de campaña del presidente Uribe como las políticas del nuevo gobierno, las recomendaciones de la Misión del Ingreso Público y las expectativas de gremios y demás fuerzas influyentes. La discusión empezó a girar en torno a una vieja discusión relacionada con la teoría de la redistribución de la riqueza y la regresividad o no de ciertos tributos, en concreto, las bondades de la tributación directa vs la tributación indirecta.

El gobierno comenzó un proceso de reestructuración de las finanzas públicas, consolidándose a finales de 1999 con el paquete de ayuda proveniente del Fondo Monetario Internacional. A raíz de esta reestructuración, se ha venido presentando un ajuste fiscal desde 2000. Colombia para promover el proceso de paz en el contexto de la implementación del "Plan Colombia" necesitaba gastar cerca de 0,3 $\%$ del PIB (2002). El gasto adicional refleja el esfuerzo del gobierno en estas áreas ${ }^{18}$.

\footnotetext{
${ }^{18}$ La continuación del proceso de paz fortalecerá la posición económica del país en el mediano plazo, pero requiere un aumento temporal en el gasto para su adecuada implementación: Los gastos adicionales se emplearían para inversión social en zonas de conflicto, fortalecimientos de la capacidad de las fuerzas armadas y refuerzo de actividades de control del narcotráfico...Después de tener presente
} 


\section{Balance Fiscal Sector Público Consolidado, 2000-2010}

A comienzos del periodo de gobierno de Uribe, el déficit del sector público consolidado (SPC) pasó de 3,4\% del PIB en el 2000; a 3,3\% al cerrar el año 2001, mostrando una mejoría del 0.1\% del PIB. Esta mejoría se explica por una mejoría en el déficit del sector público no financiero (SPNF) de $0,1 \%$ del PIB y de la mejoría en el balance del superávit del Banco de la República (ver tabla 1)

Tabla 1. Balance fiscal sector público consolidado (2000-2001)

\begin{tabular}{|l|c|c|c|c|}
\hline \multicolumn{1}{|c|}{ Balances por Período } & \multicolumn{2}{c|}{ \$ Miles de millones } & \multicolumn{2}{c|}{$\%$ PIB } \\
\hline & 2000 & 2001 & 2000 & 2001 \\
\hline 1. Sector Público No Financiero & $-6,567$ & $-7,142$ & $-3,9$ & $-3,8$ \\
\hline Gobierno Nacional Central & $-10,082$ & $-10,774$ & $-5,9$ & $-5,8$ \\
\hline Sector Descentralizado & 3,515 & 3,632 & 2,1 & 1,9 \\
\hline 2. Balance cuasifiscal del Banrep. 2/ & 877 & 1,393 & 0,5 & 0,7 \\
\hline 3. Balance de Fogafín & 30 & 327 & 0,7 & 0,2 \\
\hline 4. Costo de la Restructuración Financiera & -666 & $-1,268$ & $-0,4$ & $-0,7$ \\
\hline 5. Discrepancia Estadistica & 544 & 593 & 0,3 & 0,3 \\
\hline SECTOR PUBLICO CONSOLIDADO & -5782 & -6097 & $-3,4$ & $-3,3$ \\
\hline
\end{tabular}

Fuente: Minhacienda (2009)

La reforma fiscal, que contó con la participación activa del Presidente de la República y del Congreso, fue aprobada en diciembre de 2002 (Ley 788 de 2002). También se aprobó la reforma pensional (Ley 797 de 2002) y se avanzó en la reforma del Estado. Por otro lado, la aprobación de la Ley de responsabilidad fiscal se aplazó un semestre y fue aprobada finalmente en 2003 (Ley 819 de 2003). La reforma tributaria tendría un incremento permanente en el recaudo cercano al $2 \%$ del PIB, y el conjunto de re tendría un impacto de cerca de cuatro puntos para finales de la década de 2000, que era lo recomendado por la Misión del Ingreso Público (Junguito, 2003).

En el período 2002-2003, el balance del sector público consolidado arrojo un resultado en el déficit del sector público no financiero (SPNF) del 3,8\% del PIB en el 2002 a 3.0\% del PIB para el 2003, mostrando una reducción del 0,8\% del PIB a finales del período, lo que incidió en el mejoramiento del déficit del sector público consolidado.

estos gastos, el déficit del sector público combinado alcanzará 2,6\% del PIB (2002)(ver http://www.banrep.gov.co/documentos /publicaciones/pdf/Fmi\%20-inten). 
En la siguiente tabla se muestra el balance del Sector Público Consolidado, el cual paso de 1,3\% del PIB en el 2004 a 0,0\% en el 2005. Esta mejoría en el déficit se explica principalmente por el comportamiento del Sector Público no Financiero (SPNF), donde las variaciones más importantes se observan en el Gobierno Nacional Central.

Tabla 2. Balance fiscal sector público consolidado (2002-2003)

\begin{tabular}{|l|c|c|c|c|}
\hline \multicolumn{1}{|c|}{ Balances por Período } & \multicolumn{2}{c|}{ \$Miles de millones } & \multicolumn{2}{c|}{$\%$ PIB } \\
\hline 1. Sector Público No Financiero & 2002 & 2003 & 2002 & 2003 \\
\hline Gobierno Nacional Central & $-7,798$ & $-6,705$ & $-3,8$ & $-3,0$ \\
\hline Sector Descentralizado & $-12,434$ & $-12,151$ & $-6,1$ & $-5,4$ \\
\hline 2. Balance cuasifiscal del Banrep. 2/ & 4,636 & 5,447 & 2,3 & 2,4 \\
\hline 3. Balance de Fogafín & 1,635 & 1,437 & 0,8 & 0,6 \\
\hline 4. Costo de la Restructuración Financiera & 601 & 582 & 0,3 & 0,3 \\
\hline 5. Discrepancia Estadística & $-1,181$ & -941 & $-0,6$ & $-0,4$ \\
\hline SECTOR PUBLICO CONSOLIDADO & -627 & -795 & $-0,3$ & $-0,4$ \\
\hline
\end{tabular}

Fuente: Minhacienda (2009)

Por su parte, el Gobierno nacional central (GNC) registró un déficit equivalente a 4,9\% del PIB. Frente al año 2004 esta cifra revela una reducción en el tamaño del déficit, asociada principalmente con el dinamismo de los ingresos. En términos anuales, las rentas totales aumentaron 14,8\% y los gastos 10,3\% (Restrepo, 2005). Es el déficit más bajo de los años anteriores, y obedece a un importante crecimiento de los ingresos tributarios, debido en parte al efecto de la revaluación sobre el crecimiento de las importaciones que fortaleció el recaudo por IVA externo y por aranceles, y al aumento de la actividad económica que se reflejó en más recaudo por impuestos internos. De igual manera, a pesar de la rigidez de algunos gastos, los gastos corrientes se mantuvieron bajo control, su crecimiento entre 2004 y 2005 fue de 5\%, reflejando el compromiso de ajuste fiscal del Gobierno Nacional Central.

Tabla 3. Balance fiscal sector público consolidado (2004-2005)

\begin{tabular}{|l|c|c|c|c|}
\hline \multicolumn{2}{|c|}{ Balances por Período } & \multicolumn{2}{c|}{ \$ Miles de millones } & \multicolumn{2}{c|}{ \% PIB } \\
\hline & $\mathbf{2 0 0 4}$ & $\mathbf{2 0 0 5}$ & $\mathbf{2 0 0 4}$ & $\mathbf{2 0 0 5}$ \\
\hline 1. Sector Público No Financiero & $\mathbf{- 3 5 8 6 , 0}$ & $\mathbf{- 9 8 8 , 0}$ & $\mathbf{- 1 , 4}$ & $\mathbf{- 0 , 3}$ \\
\hline Gobierno Nacional Central & $-13983,0$ & $-13730,0$ & $-5,5$ & $-4,8$ \\
\hline Sector Descentralizado & 10396,0 & 12742,0 & 4,1 & $-4,5$ \\
\hline Seguridad Social & 4009,0 & 5844,0 & 1,6 & $-2,1$ \\
\hline Empresas del nivel nacional & 2207,0 & 3701,0 & 0,9 & $-1,3$ \\
\hline Empresas del nivel local & 1349,0 & 1044,0 & 0,5 & $-0,4$ \\
\hline Gobiernos Regionales y Locales $1 /$ & 2832,0 & 2153,0 & 1,1 & $-0,8$ \\
\hline
\end{tabular}




\begin{tabular}{|l|c|c|c|c|}
\hline 2. Balance cuasifiscal del Banrep. 2/ & 1225,0 & 669,0 & 0,5 & $-0,2$ \\
\hline 3. Balance de Fogafín & 720,0 & 610,0 & 0,3 & $-0,2$ \\
\hline 4. Costo de la Restructuración Financiera & $-920,0$ & $-1233,0$ & $-0,4$ & $-0,4$ \\
\hline 5. Discrepancia Estadistica & $-886,0$ & 919,0 & $-0,3$ & 0,3 \\
\hline SECTOR PUBLICO CONSOLIDADO & $-3447,0$ & $-24,0$ & $-1,3$ & 0,0 \\
\hline
\end{tabular}

Fuente: Minhacienda (2009)

El balance fiscal del Sector Público Consolidado (SPC) en el año 2007 mostro un déficit de \$2.714 $\mathrm{mm}$, equivalente a $0,8 \%$ del PIB. Hay que destacar que este resultado es inferior a la meta inicial establecida para 2007 (1,3\% del PIB) y levemente superior al proyectado en el marco Fiscal de Mediano Plazo 2007 (0.7\% del PIB). Por su parte, el superávit primario del SPNF disminuyó de 3,8\% del PIB en 2006 a 3,5\% del PIB en 2007 mientras la deuda pública neta de activos financieros se situó en un nivel de $27,1 \%$ del PIB (ver tabla 4 ).

Tabla 4. Balance fiscal sector público consolidado (2006-2007)

\begin{tabular}{|l|c|c|c|c|}
\hline \multicolumn{2}{|c|}{ Balances por Período } & \$ Miles de millones & \multicolumn{2}{c|}{$\%$ PIB } \\
\hline & $\mathbf{2 0 0 6}$ & $\mathbf{2 0 0 7}$ & $\mathbf{2 0 0 6}$ & $\mathbf{2 0 0 7}$ \\
\hline 1. Sector Público No Financiero & $\mathbf{- 3 , 2 0}$ & $\mathbf{- 4 , 2 1}$ & $\mathbf{- 1 , 0}$ & $\mathbf{- 1 , 2}$ \\
\hline Gobierno Nacional Central & $-13,07$ & $-11,51$ & $-4,1$ & $-3,2$ \\
\hline Sector Descentralizado & 9,87 & 7,29 & 3,1 & 2,0 \\
\hline Seguridad Social & 3,74 & 6,37 & 1,2 & 1,8 \\
\hline Empresas del nivel nacional & 4,25 & 1,18 & 1,3 & 0,3 \\
\hline Empresas del nivel local & 580,00 & 634,00 & 0,2 & 0,2 \\
\hline Gobiernos Regionales y Locales $1 /$ & 1,30 & $-891,00$ & 0,4 & $-0,2$ \\
\hline 2. Balance cuasifiscal del Banrep. 2/ & $\mathbf{1 , 4 4}$ & $\mathbf{1 , 6 4}$ & $\mathbf{0 , 4}$ & $\mathbf{0 , 5}$ \\
\hline 3. Balance de Fogafín & $\mathbf{7 7 3 , 0 0}$ & $\mathbf{3 1 8 , 0 0}$ & $\mathbf{0 , 2}$ & $\mathbf{0 , 1}$ \\
\hline 4. Costo de la Restructuración Financiera & $\mathbf{- 1 , 1 1}$ & $\mathbf{- 1 , 1 6}$ & $\mathbf{- 0 , 3}$ & $\mathbf{- 0 , 3}$ \\
\hline 5. Discrepancia Estadistica & $\mathbf{- 6 1 2 , 0 0}$ & $\mathbf{7 0 2 , 0 0}$ & $\mathbf{- 0 , 2}$ & $\mathbf{0 , 2}$ \\
\hline Sector Público Consolidado & $\mathbf{- 2 , 7 1}$ & $\mathbf{- 2 , 7 1}$ & $\mathbf{- 0 , 8}$ & $\mathbf{- 0 , 8}$ \\
\hline
\end{tabular}

Fuente: Minhacienda (2009)

Frente al cierre de 2008, el balance fiscal del Sector Público Consolidado (SPC) del 2009 registró una disminución equivalente a 2,7 puntos porcentuales del PIB, resultado principalmente del mayor 
déficit del Sector Público No Financiero (SPNF), que pasó de un balance superavitario de $\$ 187 \mathrm{~mm}$ (0,0\% del PIB) a un déficit de \$12.865 mm (2,6\% del PIB) en 2009.

El deterioro del SPNF se explicó tanto por el mayor déficit del GNC, como por el menor superávit del Sector Descentralizado en 0,8\% del PIB. En el caso del GNC, el balance ascendió a -4,2\% del PIB, frente a $-2,3 \%$ registrado en 2008, mientras que el Sector Descentralizado alcanzó 1,6\% del PIB, comparado con 2,4\% del PIB del año anterior (ver tabla 5).

Frente a los balances obtenidos en 2008, los componentes del sector descentralizado registraron un comportamiento opuesto. De esta manera, mientras el sector de Regionales y Locales y las Empresas del Nivel Local presentaron deterioros en sus resultados fiscales en $0,9 \%$ y $0,1 \%$ del PIB, respectivamente. Por otra parte, la Seguridad Social compenso dichos resultados, aumentando su superávit en 0,2\% del PIB, del 2008 al 2009.

En cuanto al resultado fiscal del Sector Público Financiero (SPF), vale la pena destacar el aumento en el superávit presentado por Fogafín en 0,1\% del PIB, del 2008 a 2009. Este resultado compensó, en parte, el menor balance del Banco de la República que se redujo en 0,2\% del PIB.

Adicionalmente, los costos asociados a la restructuración del sistema financiero fueron menores a los registrados en 2008 ( $\$ 1.117 \mathrm{~mm} ; 0,2 \%$ del PIB).

Tabla 5. Balance fiscal sector público consolidado (2008-2010)

\begin{tabular}{|c|c|c|c|c|c|c|}
\hline \multirow[t]{2}{*}{ Balances por Período } & \multicolumn{3}{|c|}{ \$ Miles de millones } & \multicolumn{3}{|c|}{$\% \mathrm{PIB}$} \\
\hline & 2008 & 2009 & 2010 & 2008 & 2009 & 2010 \\
\hline 1. Sector Público No Financiero & $-11067,0$ & $-12865,0$ & $-19050,0$ & $\mathbf{0 , 1}$ & $-2,6$ & $-3,5$ \\
\hline Gobierno Nacional Central & 11399,0 & $-20715,0$ & $-23381,0$ & $-2,3$ & $-4,2$ & $-4,3$ \\
\hline Sector Descentralizado & 5140,0 & 7850,0 & 4331,0 & 2,4 & 1,6 & 0,8 \\
\hline Seguridad Social & 831,0 & 6451,0 & 3826,0 & 1,1 & 1.3 & 0,7 \\
\hline Empresas del nivel nacional & 227,0 & 1002,0 & $-1504,0$ & 0,2 & 0,2 & $-0,3$ \\
\hline Empresas del nivel local & 5201,0 & $-382,0$ & $-46,0$ & 0,0 & $-0,1$ & 0,0 \\
\hline Gobiernos Regionales y Locales 1/ & 1306,0 & 779,0 & $-2055,0$ & 1,1 & 0,2 & 0,4 \\
\hline
\end{tabular}




\begin{tabular}{|l|c|c|c|c|c|c|}
\hline 2. Balance cuasifiscal del BanRep. 2/ & $\mathbf{5 0 2 1 , 0}$ & $\mathbf{5 9 9 , 0}$ & $\mathbf{2 9 9 , 0}$ & $\mathbf{0 , 3}$ & $\mathbf{0 , 1}$ & $\mathbf{0 , 1}$ \\
\hline 3. Balance de Fogafín & $\mathbf{1 3 0 6 , 0}$ & $\mathbf{9 2 6 , 0}$ & $\mathbf{9 0 1 , 0}$ & $\mathbf{0 , 1}$ & $\mathbf{0 , 2}$ & $\mathbf{0 , 2}$ \\
\hline 4. Costo de la Restructuración Financiera & $\mathbf{5 0 2 , 0}$ & $-\mathbf{1 1 1 7 , 0}$ & $-\mathbf{5 0 4 , 0}$ & $\mathbf{- 0 , 3}$ & $\mathbf{- 0 , 2}$ & $\mathbf{- 0 , 1}$ \\
\hline 5. Discrepancia Estadística & $\mathbf{1 2 7 0 , 0}$ & $\mathbf{- 1 2 3 6 , 0}$ & - & $\mathbf{- 0 , 3}$ & $-\mathbf{0 , 2}$ & - \\
\hline SECTOR PUBLICO CONSOLIDADO & $\mathbf{6 7 8 , 0}$ & $\mathbf{- 1 3 6 9 5 , 0}$ & $\mathbf{- 1 8 3 5 4 , 0}$ & $\mathbf{- 0 , 1}$ & $\mathbf{- 2 , 8}$ & $\mathbf{- 3 , 4}$ \\
\hline
\end{tabular}

Fuente: Minhacienda (2009)

Según PROEXPORT, a partir de 2004 diferentes factores internos y externos continúan favoreciendo el crecimiento económico de Colombia. Entre los factores internos sobresalieron la mayor dinámica de la inversión, que fue el componente de la demanda que más contribuyó al crecimiento económico en 2004; las exportaciones que, presentaron el mayor crecimiento anual de los últimos 10 años; las tasas de interés que se ubicaron en el nivel más bajo desde 1992; la menor tasa de inflación de los últimos 30 años; el déficit fiscal más bajo desde 1999, y la mejora en las condiciones de seguridad gracias a la política de defensa y seguridad democrática del gobierno.

El Actos Legislativo 01 de 2001 que, por mandato del FMI, desligó las transferencias de los ingresos corrientes de la Nación, que en vez de valer el 6,5\% del PIB, para el 2007 llegaban solo al 5,2\% del PIB. Como resultado de esta reforma y la Ley 715, el monto total asignado a las regiones, en pesos de 2006, disminuyó para ese año de 19,8 billones a 16,4 billones, generando una pérdida real alrededor de tres billones y medio. Con la de 2007, para el 2009 solamente para educación se dejaría de girar un billón y medio adicional. De esto, las consecuencias más funestas resultan ser "el deterioro de un conjunto de indicadores" el de la salud pública, agravada por el sistema de intermediación financiera en el sistema creado desde la Ley 100 de 1993 (pensiones). En el sistema educativo, “significó la disminución en cupos para la educación media y básica y, de acuerdo con Rodríguez, su impacto se verá en los programas de cobertura, pero sobre todo en la calidad, aspecto que se ha convertido en la mayor tragedia del sistema" (CID, 2007, pp. 129-183).

Con el apoyo de la Nación se reestructuraron créditos por \$1.8 billones Gracias al mejoramiento de la economía por la política de seguridad democrática, y a la nueva sobretasa a la gasolina, la cesión del IVA de licores importados a los departamentos, el cobro por grado alcoholimétrico a los aperitivos, se fortalecieron los ingresos tributarios de las Gobernaciones y las Alcaldías, los cuales aumentaron de 
$\$ 5.9$ billones en 2002 (2.9\% del PIB) a $\$ 7.9$ billones en 2004 (3.1\% del PIB) y se proyectan en $\$ 8.5$ billones para el 2005. 18 departamentos y 92 municipios en el programa de saneamiento fiscal y financiero (ver Ley 617 de $2000^{19}$ y Ley 550 de $1999^{20}$ ).

La política fiscal del gobierno de Álvaro Uribe se ha venido poco a poco consolidando sobre el trasfondo del deterioro del sector agrario y de la disminución en la calidad de vida. La deuda externa crece a pasos agigantados colocando el Producto Interno y el Presupuesto a su servicio, junto con el gasto en seguridad y el fortalecimiento de la Fuerza Pública. Las multinacionales, la banca internacional al igual que los grandes conglomerados mantienen su posición de privilegio en ganancias y fortunas. De la misma manera ocurre con los grandes neolatifundistas que han consolidado su contrarreforma agraria

Esta política se debe a que el mejoramiento de la situación fiscal ha sido uno de los requisitos clave exigidos por las agencias calificadoras, para devolver al país el grado de inversión de su deuda, perdido durante 1999, con el cual el país puede tener acceso al mercado de capitales. Por otro lado, el Ministerio de Hacienda manifestó que se seguirían realizando operaciones de manejo de deuda, para reducir tasas de interés y ampliar plazos, teniendo en cuenta el mayor recaudo tributario y la mayor disponibilidad de caja.

El crecimiento económico logrado por el país durante este período, ha permitido que el gobierno recaude impuestos a niveles muy por encima de sus metas, lo cual alivia la presión de ir a buscar mayor endeudamiento en el mercado local e internacional para financiar su gasto.

Por otro lado, es necesario resaltar que lo realmente importante no es el valor del déficit, sino la forma como éste se financia. Igualmente, y como fue analizado anteriormente, la constitución de 1991 trajo desequilibrios por lo que una forma de mejorar varios puntos de presentaría en forma de reforma

\footnotetext{
${ }^{19}$ Por la cual se reforma parcialmente la Ley 136 de 1994, el Decreto extraordinario 1222 de 1986, se adiciona la Ley Orgánica de Presupuesto, el Decreto 1421 de 1993, se dictan otras normas tendientes a fortalecer la descentralización, y se dictan normas para la racionalización del gasto público nacional.

${ }^{20}$ Con la aplicación de la Ley 550 de 1999 a las entidades territoriales, se persiguen los siguientes fines:

1. Restablecer la capacidad de pago de las entidades de manera que puedan atender adecuadamente sus obligaciones,

2. Procurar una óptima estructura administrativa financiera y contable de las mismas una vez reestructuradas,

3. Propender porque las empresas y sus trabajadores acuerden condiciones especiales y temporales en materia laboral que faciliten su reactivación y viabilidad

4. Facilitar la garantía y el pago de los pasivos pensiónales a cargo de las entidades del orden territorial
} 
constitucional, estableciendo además establecimiento un marco que apoye la restricción presupuestal cuantitativa y que asegure la adecuada recaudación de impuestos, entre otros., que deben buscar el bien común y asegurar el correcto manejo del tesoro público, teniendo en cuenta que éste pertenece a todos y cada uno de los ciudadanos del país.

Evaluar el régimen de transferencias para hacerlo más efectivo, de manera que se pueda otorgar autonomía total a las entidades territoriales en el uso de estos recursos.

En cuanto a este último punto, es preciso indicar que gran parte del déficit surge del compromiso político que le desembolsa recursos a las regiones, mas no exige compromisos y responsabilidades de suplir el gasto del gobierno central. Se inhibe parte de los impuestos a las importaciones, pero se remedia por un nuevo recaudo tributario bastante mayor que eleva las tarifas de todos los impuestos y las contribuciones a la seguridad social. De acuerdo a esto, en cierta medida es cierta la afirmación del candidato Enrique Peñalosa que dice que los contribuyentes urbanos subsidian el gasto de las regiones.

Según Salomón Kalmanovitz, ex codirector del Banco de la República, “dentro del gobierno siempre existe un pretexto para poseer déficits fiscales que radica en colgarles el sobrenombre de estructurales". Así, se afirma que el déficit fiscal no es tanto una decisión de los gobiernos como un fenómeno estructural generado por un sistema fiscal regresivo por serias fallas en la planeación y gestión del Estado.

Durante los puntos tratados anteriormente, se conoce que variables como la inflación, la tasa de interés e incluso la tasa de cambio han mostrado estabilidad a partir del establecimiento de la política económica de Uribe Vélez. Es de reconocer que la baja en el déficit fiscal logrado para todo el sector público es un logro importante, pero aún se están viviendo las consecuencias sociales que éste fenómeno ha traído.

Sin duda, el prolongado conflicto armado afecta el ejercicio económico, principalmente según expertos, a través de la acumulación de capital, y en Colombia lo que se ha rezagado de manera notoria ha sido la productividad multifactorial. Es necesario también anotar que el conflicto armado es de vieja data y que de una u otra forma el país ha ido acomodando su evolución económica al desarrollo de la lucha armada, es claro que este toma matices diferentes con cada gobierno, y el de Uribe no ha sido la 
excepción, dado que se han registrado aumentos en el gasto militar y se han presentando hechos como el debilitamiento de los grupos de narco guerrillas y episodios de parapolítica, que afectan la imagen del gobierno, y por lo tanto su credibilidad en todos los aspectos.

El problema del terrorismo, el desplazamiento, el narcotráfico, la deuda pública, el índice de desempleo, la crisis del sector industrial y comercial, el desmejoramiento de calidad de vida, demuestran que Colombia es hoy un país que precisa de una transformación de su sistema político para lograr una óptima injerencia del Estado, que busque afianzar y ajustar la economía del país.

\section{CONCLUSIONES}

Este documento ha pretendido realizar un análisis del rol del Estado en la Economía y de forma cronológica la política fiscal en Colombia durante el período 1990-2010, inicio de la apertura económica del país y finalización de la administración del presidente Álvaro Uribe Vélez, haciendo énfasis en los aspectos económicos y sociales que implicaros los aciertos y las dificultades fiscales por los que ha pasado el país.

Referente a los efectos fiscales de la Constitución de 1991, varios estudios han concluido que aumentó descomunalmente el gasto público y las transferencias, generó más déficit fiscal, estableció inflexibilidades estructurales que han hecho imposible para el país el lograr un balance fiscal sostenible y asegurar un crecimiento similar al histórico. Por los que a mayor déficit, es necesaria la búsqueda de fuentes de financiamiento interno y externo para cubrir la falta de dineros, pero un aumento de la deuda se traduce en aumento de los intereses, lo cual se traduce en problema mayor, ya que se muestra como estructural y parece no tener una salida clara.

Entre 1990 y 2003 el gasto del Gobierno en lo que respecta a su participación en el PIB ha aumentado, lo que es explicado principalmente por el comportamiento de las transferencias territoriales, pensiones y los intereses de la deuda.

Las reformas presupuestales no han tenido el éxito esperado, en términos de flexibilizar y reducir el gasto público en Colombia. 
Una de las principales causas del desequilibrio fiscal es la debilidad estructural del Estado, caracterizado por ser ineficaz, corrupto y precario, incapaz de asumir y resolver los problemas por los que atraviesa el país. El Estado colombiano es incapaz de tomar decisiones propias; sus políticas son de corto plazo, influidas por organismos multilaterales o, en su defecto, por grupos de insurgencia.

Para resolver el problema fiscal y encauzar la economía por un nuevo sendero de crecimiento requiere cambios profundos en la política económica. En primer lugar, una reprogramación de la deuda externa, de forma que su servicio sea menos oneroso para el fisco, un pacto fiscal que permita modificar el cálculo de las transferencias a los entes territoriales, y en tercer lugar, una acción decidida del Gobierno y el Congreso para combatir la evasión y la elusión, y sobre todo la eliminación de las exoneraciones que benefician las rentas de capital.

\section{REFERENCIAS}

Alemán Brizuela, C. (2002). El impacto de la política fiscal en la redistribución del ingreso en El Salvador en el año 2002. Disponible http://www.uca.edu.sv/deptos/economia/media/archivo/aefa02_impactodelapoliticafiscalenlaredist ribucion.pdf

Banco de la República. Acuerdo extendido de Colombia con el FMI. Disponible en: www.banrep.gov.co/publicaciones/pub_fmi.htm

Centro de Investigaciones para el Desarrollo (2007). Bien-estar y macroeconomía: más allá de la retórica, Bogotá, Universidad Nacional de Colombia.

Clavijo-Vera, S., Vera-Sandoval, A., Vera-Concha, N. (2015). Politica monetaria: reglas y discreción. Panorama Económico, 23, 31-38. DOI: https://doi.org/10.32997/2463-0470-vol.23-num.1$\underline{2015-1375}$

De la Hoz, A., Camargo-Montoya, P., Saldarriaga-Arango, C., Praj, D. (2014). Análisis de los riesgos en las importaciones colombianas. Panorama Económico, 22, 63-75. DOI: https://doi.org/10.32997/2463-0470-vol.22-num.0-2014-1360 
Departamento Nacional de Planeación (1970). Plan de desarrollo en marcha. Bogotá: Tercer Mundo, 1970.

Departamento Nacional de Planeación (2008). Avances y retos de la política social en Colombia.

Departamento Nacional de Planeación (1995). El Salto Social: plan nacional de desarrollo 1994-1998, Bogotá, Presidencia de la República.

Flores-Tapia, C., Flores-Cevallos, K. (2017). Impactos económicos inducidos por la demanda final en sectores estratégicos para el cambio de la estructura productiva del Ecuador. Panorama Económico, 25, 3, 443-458. DOI: https://doi.org/10.32997/2463-0470-vol.25-num.3-2017$\underline{2085}$

García-García, F. (2017). Debate sobre la inclusión del cooperativismo dentro de las políticas de la nueva ruralidad en América Latina. Panorama Económico, 25, 3, 333-356. DOI: https://doi.org/10.32997/2463-0470-vol.25-num.3-2017-2081

Junguito, R. (2003). Memorias del Ministerio de Hacienda y Crédito Público 1984-1995, Bogotá, Banco de la República.

Lozano, I. (2009). Caracterización de la política fiscal en Colombia y análisis de su postura frente a la crisis internacional. Bajado de http://www.banrep.gov.co/docum/ftp/borra566.pdf

Missaglia, M. (2015). ¿Se pueden aplicar las ideas Keynesianas Al largo plazo? unas reflexiones teóricas y un modelo ilustrativo. Panorama Económico, 23, 17-30. DOI: https://doi.org/10.32997/2463-0470-vol.23-num.1-2015-1380

Ocampo, J. (1997). Memorias al Congreso Nacional, 1996 y 1997, Bogotá. Ministerio de hacienda y Crédito Público. 
Perry, G. (1995). Memoria al Congreso Nacional 1994-1995, Bogotá, Ministerio de Hacienda y Crédito Público, Editextos 2000 Ltda.

Restrepo, J. C. (1999). Memoria de Hacienda 1998-1999, Bogotá, Imprenta Nacional de Colombia.

Restrepo, J. C. (2000). Memoria de Hacienda 1999-2000, Bogotá, Imprenta Nacional de Colombia.

Reyes, F. (2007). Reflexiones críticas al proyecto de acto legislativo que reforma el sistema general de participaciones.

Robinson, J. y Urrutia, M. (2007). Economía colombiana del siglo XX, Fondo de Cultura Económica.

Rosas, G. (1997). Comisión de racionalización del gato y las finanzas públicas, Bogotá, Ministerio de Hacienda y DNP.

Salazar-Mosquera, G. (2017). Factores determinantes del desempeño financiero en el sector manufacturero en la República del Ecuador. Panorama Económico, 25, 2, 243-254. DOI: https://doi.org/10.32997/2463-0470-vol.25-num.2-2017-2076

Sarmiento, E. (2005). El nuevo paradigma, Bogotá, ECOE-Norma.

Smith, A. (1976). The wealth of nations. The University of Chicago Press, Chicago

Stiglitz, J. (2000). La Economía del sector público. Antoni Bosch, tercera edición.

Suárez, M. A. (2002). Modelo del FMI. Economía colombiana 1990-2000, Bogotá, Ediciones Aurora. 